\title{
Diversity and Copepods'composition off Moroccan Atlantic Coast (Northwest Africa): A Review
}

\author{
Laila El Arraj (PhD student) \\ Université Hassan II, Casablanca, Maroc \\ Institut National de Recherche Halieutique (INRH), Casablanca, Maroc \\ Ouadiaa Tazi (University Professor) \\ Université Hassan II, Casablanca, Maroc \\ Laila Somoue (PhD) \\ Karim Hilmi (PhD) \\ Mansour Serghini (PhD) \\ Omar Ettahiri (PhD) \\ Institut National de Recherche Halieutique (INRH), Casablanca, Maroc
}

doi: 10.19044/esj.2017.v13n18p272 URL:http://dx.doi.org/10.19044/esj.2017.v13n18p272

Abstract

This overview sums up the results of main investigations and knowledge about zooplankton off Moroccan Atlantic coast. Copepods diversity, spatial distribution, seasonal variability and hydrology off Moroccan Atlantic coast are given. A compilation of taxonomic list of copepods' species found therein was established from published studies, they accounted for 210. Diversity and richness varied strongly between seasons, an onshore offshore gradient was observed as well. Species composition differed from northern to southern Moroccan Atlantic coast although most dominant species off Morocco's coasts were Calanus helgolandicus, Paracalanus parvus, Acartia clausi and Corycaeus typicus. In addition, the largest number of species was found in upwelling regions. A synthesis study was established in order to spatial distribution of copepods along Moroccan Atlantic coast. The Factorial Correspondence Analysis of copepod species characterizing the most important sectors has shown different patterns of copepods distribution across Moroccan Atlantic coast; three main areas were clearly segregated according to their taxonomic composition (Northern, Central and Southern Atlantic).

Keywords: Canary Current Large Marine Ecosystem, Copepods, Hydrology, NW Africa, Zooplankton 


\section{Introduction}

Fishing industry is one of the pillars of Morocco's economy. Therefore, any factors involving this area will directly impact on this country's economy. That is why research in this field is crucial. Actually, rapid changes in the zooplankton dynamics may affect the biomass of many fish stocks (Harris, 1996). It is well-known that upwelling areas are considered as the most productive regions of the oceans (Longhurst et al., 1995, Jennings et al., 2001, Carr, 2002). Indeed, numerous studies, off Morocco and elsewhere, have shown strong relationship between upwelling activity and fish recruitment and distribution (e.g. Dickson et al., 1988, Belvèze \& Erzini, 1983, Rodriguez et al., 1999, Berraho et al., 2012).

The first zooplankton investigations off the Moroccan Atlantic coast were done by Furnestin (1957). Later, several studies about zooplankton and especially copepod's distribution were conducted within the Moroccan coastal system (Furnestin, 1976, Belfequih, 1980, Chiahou, 1990, Chiahou \& Ramdani, 1996, Chiahou, 1997, Chiahou et al., 1998, Somoue, 2004, Youssara et al., 2004, Somoue et al., 2005, Salah et al., 2012, Somoue et al., 2013, Zizah et al., 2012, Salah et al., 2013, El Arraj et al., 2015). All over the world zooplankton and copepod research has been increasing, however only a few studies (1 to 3/year) have been published about their distribution within the CCLME (Canary Current Large Marine Ecosystem) during the last two decades (Fig. 1).

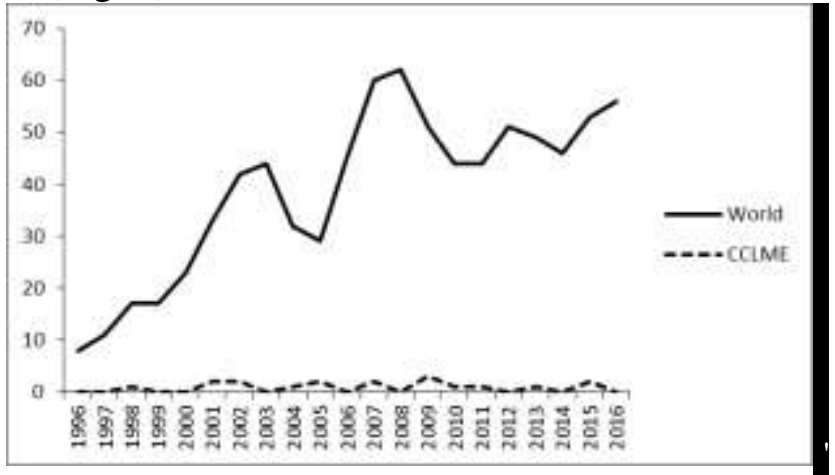

Fig. 1. Number of published papers on 'Science-direct' containing the words: zooplankton, copepod and Canary system in the title, abstract or keywords (from 1996 to 2016).

The Moroccan Atlantic coast is part of the Canary Current System (CCS) which extends between the Iberian Peninsula $\left(43^{\circ} \mathrm{N}\right)$ and the south of Senegal $\left(8^{\circ} \mathrm{N}\right)$, and dominates most hydrodynamic processes therein. Circulation along the Moroccan coast is determined by the Azores of the Saharan depression seasonal rhythm and ITCZ (Inter Tropical Convergence Zone) as well (Wooster et al., 1976, Parrish et al., 1983) (Fig. 2). 


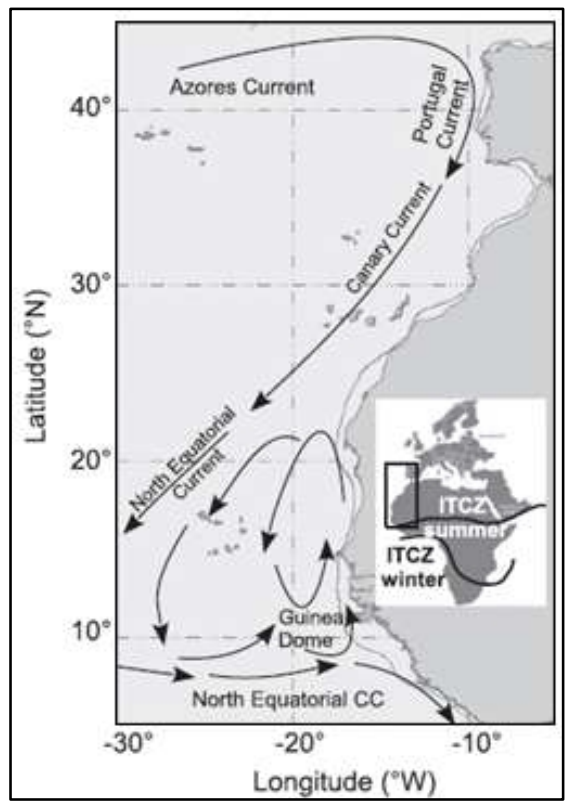

Fig. 2. Main surface currents: Azores, Portugal, Canary, North Equatorial current and North Equatorial counter-current. The $200 \mathrm{~m}$ isobath is superimposed (Benazzouz et al. 2014).

Hydrology therein is mainly submitted to the action of upwelling phenomena (e.g. Barber \& Smith, 1981, Mann \& Lazier, 1991, Cushing, 1989, Barton, 1998, Arístegui, et al. 2009). Upwelling activity along this stream is not regular and often occurs in some regions of the coast according to their topography. These areas are often close to the capes (Binet, 1988, Mittelstaedet, 1991, Ould Dedah, 1995, Longhurst, 1998). Consequently, an enhancement of nutrients is induced which leads to a significant growth of phytoplankton (Fig. 3) and zooplankton (Pauly \& Christensen, 1995). The highest densities of zooplankton are found during upwelling activity; especially near-shore (Bainbridge, 1972). 


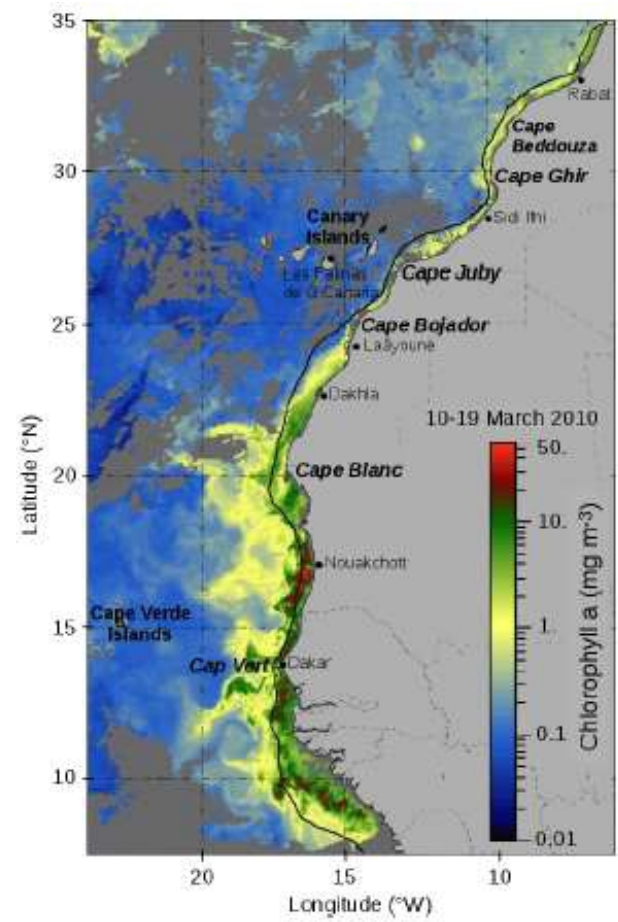

Fig. 3. Average chlorophyll-a computed from MODIS sensor data for the period 10-19 March 2010, The $200 \mathrm{~m}$ bathymetry contour (black line) is added (Demarcq \& Somoue, 2015).

Since copepods are among the most numerous multicellular organisms in the Moroccan coastal system and they represent $80 \%$ of the total biomass of plankton in the oceans (Verity \& Smetacek, 1996), special attention will be given to this group in this review. In this paper, we review the main information mentioned in the literature in order to state results about distribution and composition of mesozooplaknton; especially copepods; in Moroccan Atlantic coast. Our review provides an overview of the work conducted off the Moroccan Atlantic coast summarizing all the literature available and identifying gaps in our knowledge. This paper is not an exhaustive review of all mesozooplankton information, but we attempt to document the present state of our knowledge of this component in relation to spatial and temporal oceanographic patterns off Moroccan Atlantic coast. We particularly describe the main findings about copepods recorded until now within this area.

\section{Study area: Hydrography and upwelling}

Zooplankton abundances and community structure in coastal ecosystems are closely related to environmental changes in temperature, salinity, chlorophyll, nutrients, turbulence and particularly upwelling activity 
(Harris et al., 2000, Calbet et al., 2001, Lawrence et al., 2004, Alcaraz et al., 2007, Glushko \& Lidvanov, 2012). The pelagic resources off Atlantic Moroccan coast are mainly influenced by upwelling activity (Makaoui et al., 2005). Therefore, in order to establish distribution patterns of zooplankton in pelagic ecosystem, it is necessary to study and understand the circulation and distribution of water masses (Boltovskoy, 1999). Since the 70's several studies have dealt with the Moroccan upwelling system of Atlantic coast where Latitudinal change from perennial to seasonal upwelling is observed (e.g. Hughes \& Barton, 1974, Johnson et al., 1975, Mittelstaedt et al., 1975, Mittelstaedt \& Hamann, 1981, Mittelstaedt, 1991, Hernández-Guerra \& Nykjær, 1997, Makkaoui et al., 2005, Troupin, 2011, Makaoui et al., 2012, Laarisi et al., 2013, Benazouz et al., 2014). Makaoui et al. (2005) subdivided Moroccan Atlantic coast to four main sectors according to their upwelling activities: cape Ghir-cape Cantin $\left(30^{\circ}-33^{\circ} \mathrm{N}\right)$, cape Juby-cape Draa $\left(28^{\circ}\right.$ $29^{\circ} \mathrm{N}$ ) (characterized by an upwelling activity during summer), Dakhla-cape Bojador $\left(24^{\circ}-26^{\circ} \mathrm{N}\right)$, and cape Blanc-cape Barbas $\left(21^{\circ}-22^{\circ} \mathrm{N}\right.$ ) (upwelling activity throughout the year). Equally, Marcello et al. (2011) have identified two main upwelling areas: cape Juby-cape Ghir $\left(28^{\circ}-30^{\circ} \mathrm{N}\right.$ ) (seasonal upwelling) and cape Blanc-cape Bojador $\left(21^{\circ}-26^{\circ} \mathrm{N}\right)$ (perennial upwelling activity) which corroborate with previous studies (Mittelstaedt 1991, Hernández-Guerra \& Nykjær 1997, Pelegri et al. 2005). In fact, the distribution of planktonic organisms is chiefly determined by currents and water masses (Chiahou 1997, Cheggour 1998, Hernández-León et al. 2002, Pelegri et al. 2005). Off cape Blanc $\left(21^{\circ} \mathrm{N}\right)$, upwelling intensity shows an important variability on short scales (daily/weekly) (e.g. Mittelstaedt, 1991, Nykjær \& Van Camp, 1994, Barton, 1998, Susek, 2005) and the productivity therein is highest in boreal winter (winter) and spring. Sea-surface temperatures (SST) are low $\sim 18^{\circ} \mathrm{C}$ (Fig. 4) and yearly sea-surface salinity (36.2 psu) too (Indicating South Atlantic Central Water as the source of the upwelled water). As to sea-surface nitrate and phosphate concentrations, high values were recorded off this area; up to about $5 \mu$ mol. $\mathrm{L}^{-1}$ and 0.4 $\mu$ mol. $L^{-1}$ respectively (WOA, 2005). 

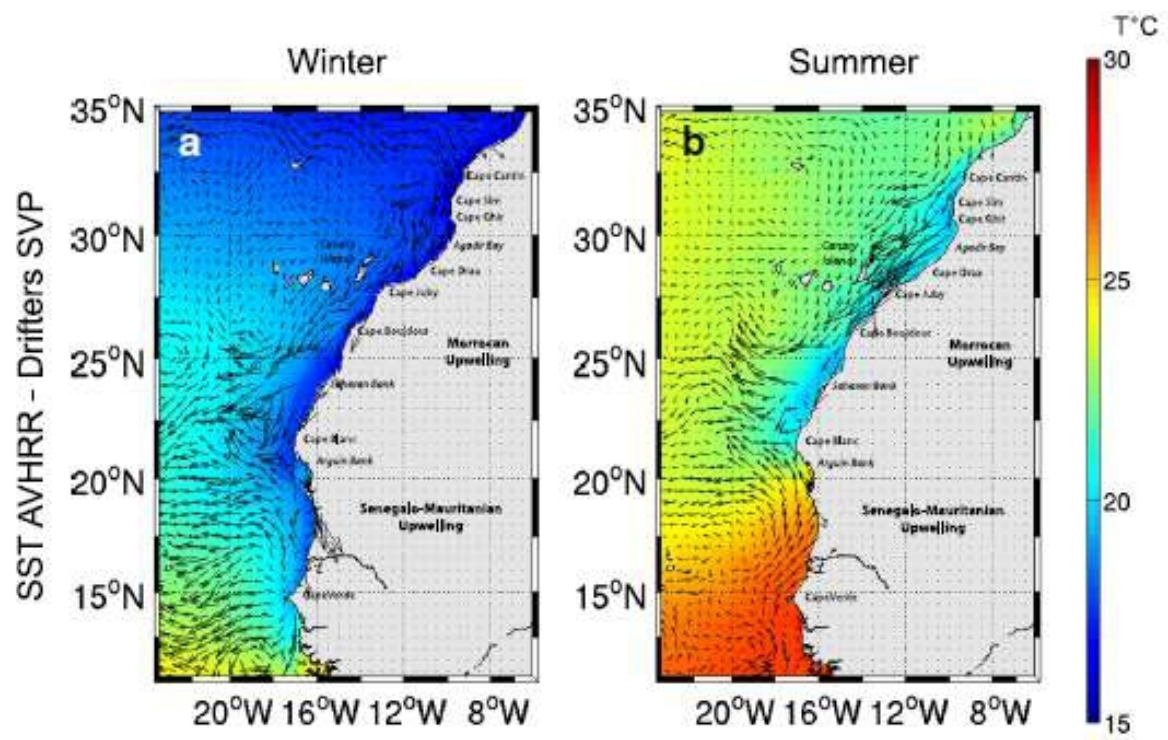

Fig. 4: Seasonal climatology of sea surface temperature (SST in background) and nearsurface currents (vectors) from AVHRR satellite data (1985-2009) and the Global Drifter Program (1979-present, Lumpkin and Johnson, 2013) in winter and summer (Auger et al. 2015).

Thus, the southern area, between Cap Blanc $\left(21^{\circ} \mathrm{N}\right)$ and cape Bojador $\left(26^{\circ} \mathrm{N}\right)$ is considered as the most productive, thanks to its intense and permanent upwelling activity (Fig. 5) (Minas et al., 1982, Binet, 1991, Makaoui et al., 2005, Benazouz et al., 2014, Cropper et al., 2014).

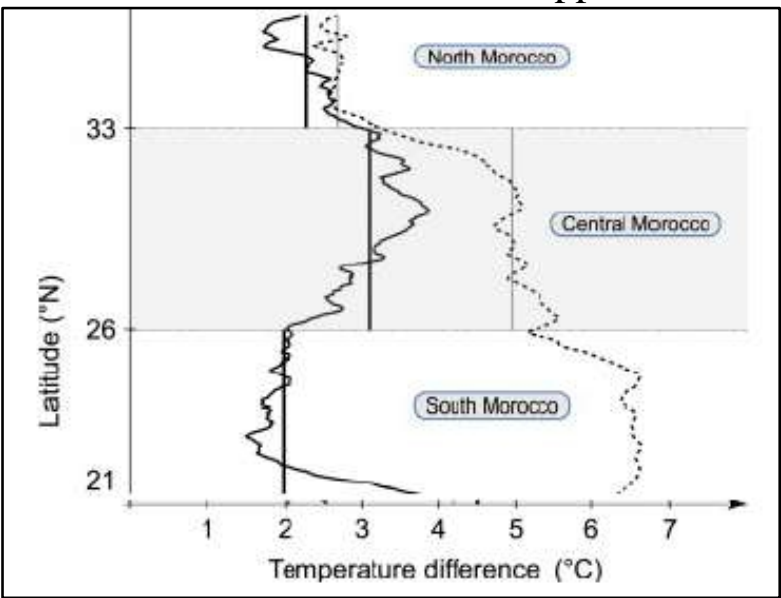

Fig. 5. Latitudinal classification of the upwelling activity off Morocco based on the average value of thermal zonal difference (discontinuous line) and its seasonal amplitude (continuous line). The vertical bars help to visualize the average values of the parameters (Modified from Benazzouz et al. 2014).

Off these two capes, intensity of upwelling is apparently increasing with global warming (Hughes \& Barton, 1974). In the region of cape Ghir 
$\left(30^{\circ} \mathrm{N}\right)$, Salah et al (2012) have used the neritic species A. clausi to follow the flow of the upwelling filament from inshore to offshore. In the same study, chlorophyll- $a$ seemed to be the major factor affecting mesozooplankton's biomass. Periods characterized by low temperature were the richest. The same results were found between cape Blanc $\left(21^{\circ} \mathrm{N}\right)$ and cape Bojador $\left(26^{\circ} \mathrm{N}\right)$ since a strong relationship was set between copepod abundance and diversity and low temperature and chl- $a$ (Somoue et al., 2005).

\section{Copepods: Specific composition and seasonal variability}

Several authors have observed dominance of copepods among zooplankton groups within the Moroccan Atlantic coast (e.g. Furnestin, 1957, Thiriot, 1978, Belfequih, 1980, Chiahou \& Ramdani, 1997, Chiahou et al., 1998, Somoue et al., 2005, Youssara et al., 2004, Salah et al., 2012, Zizah et al., 2012, Zaafa et al., 2012, Salah et al., 2013, Zaafa et al., 2014). Recently, their relative abundances were calculated across CCLME system; they are about 60-95\% of total zooplankton abundance (Berraho et al., 2015). Main copepods studies conducted off Moroccan Atlantic system are described in table 1. Although the checklist of copepods' species is from different expeditions with different sampling methods, all of them were focusing on mesozooplankton $(>160-200 \mu \mathrm{m})$.

In our study, 210 species of copepods were recorded off the Moroccan Atlantic coast according to data collected from literature (see table 2). Recently, copepods composition has been reviewed within the same study area (cape Spartel $36^{\circ} \mathrm{N}$-cape Blanc $21^{\circ} \mathrm{N}$ ) by Berraho et al. (2015) who reported 104 species; 103 of these species found among species listed on table 2 except the species Clausocalanus parapergens.

Within the Moroccan continental shelf, several authors have recorded higher diversity offshore than inshore stations (e.g. Grall et al., 1974, Belfequih, 1980, Boucher, 1987, Somoue, 2004, Salah et al., 2012, El Arraj et al., 2015) which is probably due to the extension of the filaments generated from Canary Current which are born near the capes of African coast since they are a result of the interaction of the current with the coastal cape morphology (Hagen et al., 1996, Hernández-Guerra \& Nykjær, 1997, Barton et al., 1998, Stevens \& Johnson, 2003). They could extend for hundreds of kilometers offshore at pronounced capes like cape Ghir $\left(30^{\circ} \mathrm{N}\right)$, cape Juby $\left(28^{\circ} \mathrm{N}\right)$ and cape Blanc $\left(21^{\circ} \mathrm{N}\right)$ (Van Camp et al., 1991, Nykjær \& Van Camp, 1994, Hernández-Guerra \& Nykjær, 1997, Hagen, 2001).

Somoue (2004) has noted greater richness within the southern region of Dakhla $\left(24^{\circ} \mathrm{N}\right)$ than in the northern area $\left(26^{\circ}-24^{\circ} \mathrm{N}\right)$.

Earlier, Furnestin (1957) has found important abundances during autumn along Moroccan Atlantic shore. According to Somoue (2004), in 
winter, higher copepods densities (3-183 ind $\mathrm{m}^{-3}$ ) were observed within latitudes $21^{\circ} \mathrm{N}, 22^{\circ} 30 \mathrm{~N}, 23^{\circ} 30 \mathrm{~N}, 25^{\circ} \mathrm{N}$ and $26^{\circ} \mathrm{N}$. During summer, their densities were relatively low $\left(2-83\right.$ ind. $\left.\mathrm{m}^{-3}\right)$ except in cape Blanc $\left(21^{\circ} \mathrm{N}\right)$ and cape Barbas $\left(22^{\circ} \mathrm{N}\right)$ whereas in the northern part of Morocco, high abundances were recorded within inshore stations, particularly during warm seasons (spring and summer) compared to autumn (Zaafa et al., 2012, 2014).

Generally, copepods are more abundant during hot seasons within several studied areas along Moroccan Atlantic coast (Belfequif, 1980, Chiahou \& Ramdani, 1996, Zizah et al., 2012, Salah et al., 2013, Somoue et al., 2013). Actually, their seasonal variability is closely related to hydrological characteristics and phytoplankton dynamic while inter-annual variability is apparently related to atmospheric circulation (Valdés \& Moral, 1998). By contrast, diversity and richness were higher during cold seasons coinciding with upwelling activities (e.g. Chiahou \& Ramdani, 1997, Somoue et al., 2005, Zaafa et al., 2010, Salah et al., 2013, Somoue et al., 2013).

\section{Synthesis study}

In order to illustrate geographical distribution of copepod species, we have selected upwelling areas of which data are available [(cape Spartel $\left(36^{\circ} \mathrm{N}\right)$, cape Ghir $\left(30^{\circ} \mathrm{N}\right)$, cape Juby $\left(28^{\circ} \mathrm{N}\right)$, cape Bojador $\left(26^{\circ} \mathrm{N}\right)$, cape Barbas $\left(22^{\circ} \mathrm{N}\right)$ and cape Blanc $\left.\left(21^{\circ} \mathrm{N}\right)\right]$. A Factorial Correspondence Analysis (FCA) was established between total species recorded within the six areas based on binary matrix (presence-absence) to visualize preferential geographic distribution of the species recorded within these areas. Although the campaigns were carried out during different seasons and conducted using different methods, this analysis had a descriptive objective ignoring sampling conditions (Fig. 6). 


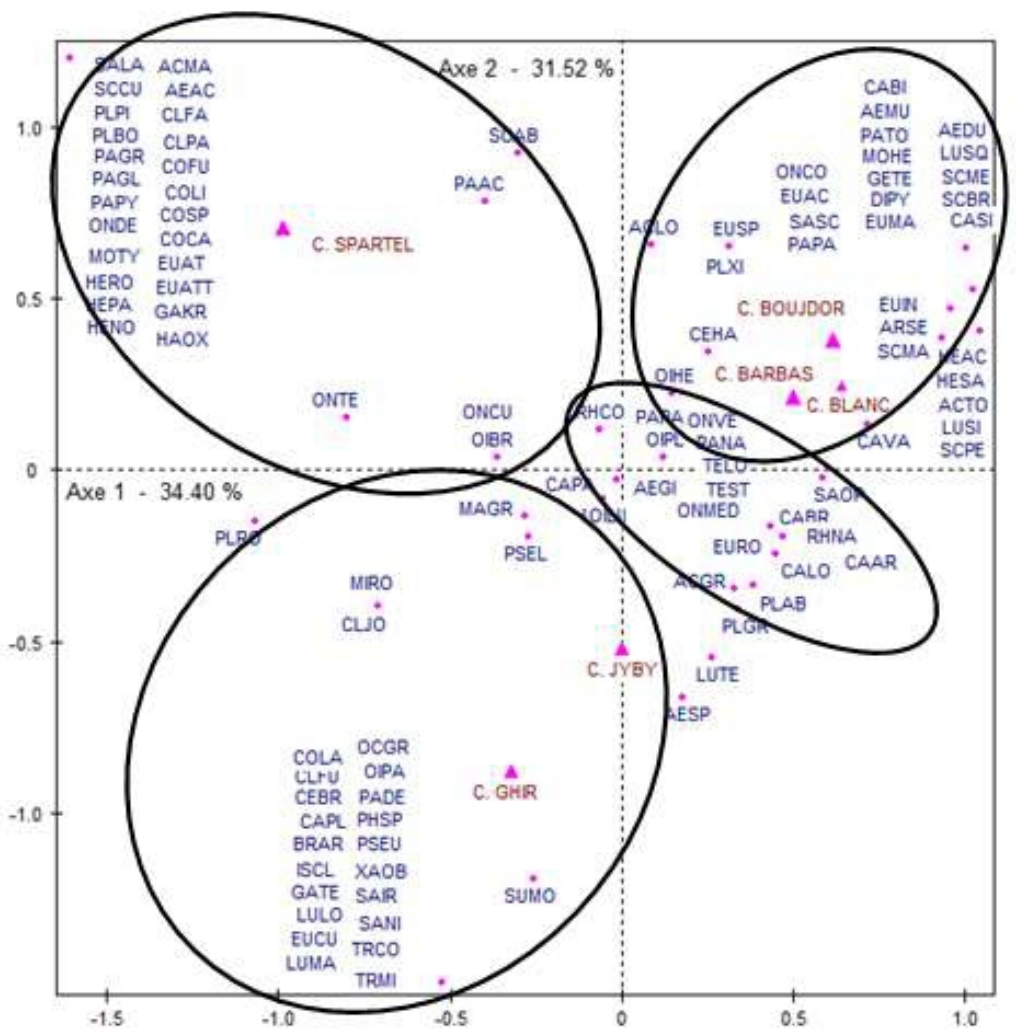

|Fig. 6. FCA (Factorial Correspondence Analysis) of total species reported within Cape Spartel $\left(36^{\circ} \mathrm{N}\right)$, Cape Ghir $\left(30^{\circ} \mathrm{N}\right)$, Cape Juby $\left(28^{\circ} \mathrm{N}\right)$, Cape Bojador $\left(26^{\circ} \mathrm{N}\right)$, Cape Barbas $\left(22^{\circ} \mathrm{N}\right)$ and Cape Blanc $\left(21^{\circ} \mathrm{N}\right)$ using the software ADE4 (Descriptive Analysis Ecological: methods Exploratory and Euclidean in Environmental Sciences). "Four capital letters indicate species name of dominant species at each region (cf. Table 2)".

As a result, the factorial plan F1XF2 presented $65.92 \%$ of total inertia at the rate of $34.40 \%$ for $\mathrm{F} 1$ axis and $31.52 \%$ for $\mathrm{F} 2$ axis. Therefore, the projection of species on the factorial plane reveals a segregation of 4 distinguished groups of species. The first assemblage that includes only the three capes of the southern area: cape Bojador $\left(26^{\circ} \mathrm{N}\right)$, cape Barbas $\left(22^{\circ} \mathrm{N}\right)$ and cape Blanc $\left(21^{\circ} \mathrm{N}\right)$. These three regions have in common a number of species which are for the most of them tropical to sub-tropical origin as $L$. squillimana, A. tonsa (Owre \& Foyo, 1967). In addition, this group harbors the highest number of species. The second group included species of both capes of the central studied area (cape Ghir $30^{\circ} \mathrm{N}$ and cape Juby $28^{\circ} \mathrm{N}$ ). Most of the species recorded therein have been qualified as predominant within the canary current system as C. pavo, S. opalina, L. tenuicauda (Lozano Sedovilla et al., 1988, Chiahou \& Ramdani, 1998) whereas the region of cape Spartel $\left(36^{\circ} \mathrm{N}\right)$ was individualized harboring Mediterranean, Lusitanian and boreal species as A. margalefi, P. borealis, P. gracilis, M. typical, G. 
kruppi and C. limbatus (Mazza, 1967, Coen \& Mazzocchi, 1985, Chiahou \& Ramdani, 1998). According to Chiahou \& Ramdani (1998), the faunistical exchange between the Mediterranean Sea and Atlantic Ocean is apparently easier than the exchange between the Moroccan Atlantic region and the Ibero-French area. Deep currents, bringing Mediterranean waters to Atlantic Ocean seems to have its maximum flow during summer and autumn (Furnestin, 1957). The fourth group contained species commonly recorded within at least 4 capes. Most of these species are cosmopolitan as cited bellow like $O$. venusta, $T$. longicornis, $T$. stylifera, $P$. nanus, $P$. parvus and O. nana (Belfquih, 1980, Nishida, 1985, Razouls, 1995, Razouls, 1996).

\section{Conclusion and perspectives}

Our results suggest that species mainly dominant within the study area are $C$. helgolandicus, P. parvus, A. clausi and C. typicus; they constitute the most abundant species of coastal zooplankton communities (e.g. Seguin, 1966, Belfquih, 1980, Boucher, 1982, Vieira et al., 2003). We conclude that three distinct regions were clearly individualized by their own composition and hydrological particularities: Northern, Central and Southern area. Although Moroccan Atlantic coast is one the most productive zones in the world, the southern part (from cape Bojador $26^{\circ} \mathrm{N}$ to cape Blanc $21^{\circ} \mathrm{N}$ ) remains the richest owing to the heterogeneity of factors affecting this region; particularly to permanent upwelling activity therein. Many species considered as indicators of upwelling activity were recorded within this area like C. helgolandicus and C. carinatus (Bainbridge et al., 1960, De Decker, 1964, Seguin, 1966, Smith, 2000). Moreover, C. helgolandicus is a species widely distributed and closely related to the Canary Current System (Flenminger \& Hulseman, 1973). The central zone harbors a large number of species as well, since it is submitted to a seasonal intense upwelling activity; most of them are Atlanto-Mediterranean species; while in the northern part, this activity is weaker and harbors species brought by the intrusion of Mediterranean waters.

More comprehensive studies need to be carried out researching the regular diurnal vertical flux monitoring of copepods, the consequences of the oxygen minimum zone (OMZ) on their distribution patterns, the trophic relations and information on size spectra which should enrich and improve our knowledge of biological resources. Sampling in the huge Moroccan Atlantic coast needs to be done more regularly in order to further understand the apparent self-regulatory micro- and mesozooplankton response to environmental conditions. Performing a large scale study is necessary for a better understanding of mesozooplankton communities' evolution and distribution across this area and their response to climate change. 


\section{Acknowlegments}

We appreciate and acknowledge captains and crews of the R/Vs 'AtlantNIRO', 'Al Amir Moulay Abdellah' and 'Fridjof Nansen' for collecting samples. Special thanks go to different authors for systematic identification of copepods, spatially Oceanography department's team. This work could not have been done without the support of PPR Modelling the Moroccan Atlantic ecosystem (priority research project) between the INRH, the Faculty of Science Semlalia and the Technology School of Essaouira, funded by CNRST / Morocco.

\section{Tables}

Table 1. Overview of the selected publications related to copepods in marine waters off Moroccan Atlantic coast.

\begin{tabular}{|c|c|c|c|c|}
\hline $\begin{array}{l}\text { Study } \\
\text { Sites }\end{array}$ & Period of cruises & $\begin{array}{l}\mathrm{Nb} . \\
\text { of } \\
\mathrm{Sp}\end{array}$ & Dominant species & References \\
\hline \multirow{2}{*}{$\begin{array}{l}\text { Cape } \\
\text { Spartel } \\
\left(35^{\circ} \mathrm{N}\right)- \\
\text { Oued Draa } \\
\left(28^{\circ} 30^{\prime} \mathrm{N}\right)\end{array}$} & \multirow[t]{2}{*}{$\begin{array}{l}\text { February, May, } \\
\text { August and } \\
\text { November } 1950 .\end{array}$} & 100 & $\begin{array}{c}\text { A. clausi, } C \text {. typicus, } C . \\
\text { chierchiae, } C . \text { arcuicornis, } C . \\
\text { helgolandicus } \\
\end{array}$ & $\begin{array}{c}\text { Furnestin \& } \\
\text { Belfquih } \\
1976 . \\
\end{array}$ \\
\hline & & 103 & $\begin{array}{l}\text { A. clausi, } C \text {. chierchiae, } C . \\
\text { typicus, } C . \text { arcuicornis, } C . \\
\text { furcatus, E. acutifrons, } O . \text { nana, } \\
\text { O. similis and } P \text {. parvus. }\end{array}$ & $\begin{array}{l}\text { Belfquih } \\
1980 .\end{array}$ \\
\hline $\begin{array}{l}\text { Cape Blanc } \\
\left(21^{\circ} \mathrm{N}\right)- \\
\text { Cape Ghir } \\
\left(31^{\circ} \mathrm{N}\right) \\
\end{array}$ & $\begin{array}{c}\text { February, May, } \\
\text { August and } \\
\text { November } 1950 .\end{array}$ & 87 & $\begin{array}{c}\text { A. clausi, O. curta, O. nana, } P . \\
\text { parvus. C. jobei, T. stylifera and } \\
\text { T. longicornis. }\end{array}$ & $\begin{array}{l}\text { Boucher et } \\
\text { al. } 1982 .\end{array}$ \\
\hline $\begin{array}{l}\text { Region of } \\
\text { El Jadida } \\
\left(33^{\circ} 30^{\prime} \mathrm{N}\right)\end{array}$ & $\begin{array}{c}\text { From December } \\
1994 \text { to December } \\
1995(2-3 \\
\text { sampling/month). }\end{array}$ & 90 & $\begin{array}{l}\text { A. discaudata, A. grani and } \\
\text { Eucalanus crassus. }\end{array}$ & $\begin{array}{c}\text { Chiahou \& } \\
\text { Ramdani } \\
1996 .\end{array}$ \\
\hline $\begin{array}{l}\text { Region of } \\
\text { Agadir } \\
\left(31^{\circ} \mathrm{N}\right) \\
\end{array}$ & $\begin{array}{l}\text { From May } 1999 \text { to } \\
\text { December } 2000 \text { (1 } \\
\text { sampling/15 days). }\end{array}$ & 36 & A. clausi and $P$. parvus. & $\begin{array}{l}\text { Youssara et } \\
\text { al. } 2004 .\end{array}$ \\
\hline $\begin{array}{l}\text { Cape } \\
\text { Bojador } \\
\left(26^{\circ} \mathrm{N}\right)- \\
\text { Cape Blanc } \\
\left(21^{\circ} \mathrm{N}\right)\end{array}$ & $\begin{array}{l}\text { March and July } \\
1998 .\end{array}$ & 79 & $\begin{array}{l}\text { C. helgolandicus, } P . \text { parvus, } A . \\
\text { clausi and } C \text {. typicus. }\end{array}$ & $\begin{array}{l}\text { Somoue et } \\
\text { al. } 2005 \text {. }\end{array}$ \\
\hline $\begin{array}{l}\text { Cape Ghir } \\
\left(31^{\circ} \mathrm{N}\right)\end{array}$ & $\begin{array}{c}\text { December 2008, } \\
\text { February, April, } \\
\text { June and October } \\
2009 .\end{array}$ & 86 & $\begin{array}{l}\text { O. similis, } O . \text { nana, } O . \text { venusta, } \\
\text { A. clausi, } P . \text { parvus, } E . \\
\text { acutifrons } O . \text { plumifera, } C . \\
\text { helgolandicus and } C . \\
\text { arcuicornis. } \\
\end{array}$ & $\begin{array}{l}\text { Salah et al. } \\
2012 .\end{array}$ \\
\hline $\begin{array}{c}\text { Cape } \\
\text { Bojador } \\
\left(26^{\circ} \mathrm{N}\right)- \\
\text { Cape Blanc }\end{array}$ & $\begin{array}{l}\text { June and } \\
\text { November } 2007 . \\
\text { June, November } \\
\text { and July } 2008 .\end{array}$ & 85 & $\begin{array}{c}\text { C. helgolandicus, } P . \text { parvus, } A . \\
\text { clausi and C. typicus. }\end{array}$ & $\begin{array}{l}\text { Zizah et al. } \\
2012 .\end{array}$ \\
\hline
\end{tabular}




\begin{tabular}{|c|c|c|c|c|}
\hline $\begin{array}{c}\left(21^{\circ} \mathrm{N}\right) \\
\text { Cape Juby } \\
\left(28^{\circ} \mathrm{N}\right)\end{array}$ & $\begin{array}{c}\text { February, April, } \\
\text { June and October } \\
2009 .\end{array}$ & 56 & $\begin{array}{c}\text { A. clausi, E. acutifrons, } \text {. } \\
\text { similis, } \text { O. venusta } \text { and } P . \\
\text { parvus. }\end{array}$ & $\begin{array}{c}\text { Salah et al. } \\
2013 .\end{array}$ \\
\hline $\begin{array}{c}\text { Cape } \\
\text { Spartel } \\
\left(35^{\circ} \mathrm{N}\right)\end{array}$ & $\begin{array}{c}\text { March, May and } \\
\text { December 2006. } \\
\text { May, July and } \\
\text { November 2007. }\end{array}$ & 85 & P. parvus and O. venusta.. & $\begin{array}{c}\text { Zaafa et al. } \\
2014 .\end{array}$ \\
\hline $\begin{array}{c}\text { Cape } \\
\text { Bojador } \\
\left(26^{\circ} \mathrm{N}\right)-\end{array}$ & $\begin{array}{c}\text { November 2011 } \\
\text { and July 2012. } \\
\left(21^{\circ} \mathrm{N}\right)\end{array}$ & 76 & $\begin{array}{c}\text { O. venusta, } \text { C. arcuicornus and } \\
\text { A. clause. }\end{array}$ & $\begin{array}{c}\text { El Arraj et } \\
\text { al. } 2015 .\end{array}$ \\
\hline
\end{tabular}

Table 2. Taxonomic checklist of copepod species recorded off Moroccan Atlantic coast according to studies listed above "table 1" with their respective codes (only species mentioned in "Figs. 9 and 10" are coded).

\begin{tabular}{|c|c|c|c|}
\hline Species & Code & Species & Code \\
\hline Acartia (Acartiura) clausi Giesbrecht, 1889 & ACCL & Mecynocera clausi Thompson I.C., 1888 & \\
\hline Acartia (Acartia) danae Giesbrecht, 1889 & ACDA & Megacalanus princeps Wolfenden, 1904 & \\
\hline Acartia (Acartiura) discaudata (Giesbrecht, 1881) & & Mesocalanus tenuicornis Dana, 1849 & \\
\hline Acartia (Acartiura) longiremis (Lilljeborg, 1853) & ACLO & Metacalanus inaequicornis (Sars G.O., 1903) & \\
\hline Acartia (Acartiura) margalefi Alcaraz, 1976 & ACMA & Metridia brevicauda Giesbrecht, 1889 & \\
\hline Acartia (Acartia) negligens Dana, 1849 & & Metridia lucens Boeck, 1865 & \\
\hline Acartia (Acanthacartia) tonsa Dana, 1849 & ACTO & Metridia macrura Sars G.O., 1905 & MEMA \\
\hline Aegisthus aculeatus Giesbrecht, 1891 & AEAC & Metridia princeps Giesbrecht, 1889 & \\
\hline Aegisthus mucronatus Giesbrecht, 1891 & AEMU & Metridia venusta Giesbrecht, 1889 & \\
\hline Aegisthus spinulosus Farran, 1905 & & Microcalanus pusillus Sars G.O., 1903 & \\
\hline Aetideus armatus (Boeck, 1872) & AESP & Microsetella norvegica (Boeck, 1865) & MINO \\
\hline Aetideus giesbrechti Cleve, 1904 & AEGI & Microsetella rosea (Dana, 1847) & MIRO \\
\hline Aetideopsis armatus (Boeck, 1872) & & Miracia efferata Dana, 1849 & \\
\hline Aetideopsis carinata Bradford, 1969 & & Monacilla typica Sars G.O., 1905 & MOTY \\
\hline Aetideopsis multiserrata (Wolfenden, 1904) & & Monstrilla grandis Giesbrecht, 1891 & \\
\hline Agetus limbatus (Brady, 1883) & AGLI & Monstrilla helgolandica Claus, 1863 & MOHE \\
\hline Agetus flaccus (Giesbrecht, 1891) & & Monstrillopsis dubia Scott T., 1904 & \\
\hline Agetus typicus Krøyer, 1849 & AGTY & Nannocalanus minor (Claus, 1863) & NAMI \\
\hline Anomalocera patersoni Templeton, 1837 & & Neocalanus gracilis (Dana, 1852) & NEGR \\
\hline Arietellus setosus Giesbrecht, 1893 & ARSE & Neocalanus robustior (Giesbrecht ,1888) & \\
\hline Augaptilus longicaudatus (Claus, 1863) & & Nullosetigera bidentata (Brady, 1883) & \\
\hline Augaptilus megalurus Giesbrecht, 1889 & & Oculosetella gracilis (Dana, 1849) & OCGR \\
\hline Augaptilus spinifrons Sars G.O., 1907 & & Oithona brevicornis Giesbrecht, 1891 & OIBR \\
\hline Bradyidius armatus (Vanhöffen, 1897) & BRAR & Oithona linearis Giesbrecht, 1891 & OILI \\
\hline Calanoides carinatus (Krøyer, 1849) & CACA & Oithona nana Giesbrecht, 1893 & OINA \\
\hline Calocalanus finmarchicus (Gunnerus, 1770) & CAFI & Oithona parvula (Farran, 1908) & OIPA \\
\hline Calanus hyperboreus Krøyer, 1838 & & Oithona plumifera Baird, 1843 & OIPL \\
\hline Calocalanus contractus Farran, 1926 & $\mathrm{CACO}$ & Oithona setigera (Dana, 1849) & \\
\hline Calocalanus pavo (Dana, 1852) & CAPA & Oithona similis Claus, 1866 & OISI \\
\hline Calocalanus plumulosus (Claus, 1863) & CAPL & Oncaea curta Sars G.O., 1916 & ONCU \\
\hline Calocalanus styliremis Giesbrecht, 1888 & & Oncaea media Giesbrecht, 1891 & ONME \\
\hline
\end{tabular}


Candacia armata Boeck, 1872

Candacia bipinnata (Giesbrecht, 1889)

Candacia elongata (Boeck, 1872)

Candacia ethiopica (Dana, 1849)

Candacia longimana (Claus, 1863)

Candacia simplex (Giesbrecht, 1889)

Candacia tenuimana (Giesbrecht, 1889)

Candacia varicans (Giesbrecht, 1893)

Centropages bradyi Wheeler, 1900

Centropages chierchiae Giesbrecht, 1889

Centropages hamatus (Lilljeborg, 1853)

Centropages Krøyeri Giesbrecht, 1893

Centropages typicus Krøyer, 1849

Centropages violaceus (Claus, 1863)

Chirundina streetsii Giesbrecht, 1895

Clausocalanus arcuicornis (Dana, 1849)

Clausocalanus farrani Sewell, 1929

Clausocalanus furcatus (Brady, 1883)

Clausocalanus jobei Frost \& Fleminger, 1968

Clausocalanus mastigophorus (Claus, 1863)

Clausocalanus paululus Farran, 1926

Clausocalanus pergens Farran, 1926

Clytemnestra gracilis (Claus, 1891)

Clytemnestra scutellata Dana, 1847

Copilia mediterranea (Claus, 1863)

Copilia quadrata Dana, 1849

Corycaeus clausi Dahl F., 1894

Corycaeus crassiusculus Dana, 1849

Corycaeus obtusus Dana, 1849

Corycaeus speciosus Dana, 1849

Corycaeus $s p$.

Ctenocalanus vanus Giesbrecht, 1888

Cymbasoma thompsonii (Giesbrecht, 1893)

Diaixis pygmaea (Scott T., 1894)

Ditrichocorycaeus anglicus (Lubbock, 1857)

Eucalanus elongatus Dana, 1848

Euchaeta acuta Giesbrecht, 1893

Euchaeta marina Prestandrea, 1833

Euchaeta pubera Sars G.O., 1907

Euchaeta spinosa Giesbrecht, 1893

Euchirella curticauda Giesbrecht, 1888

Euchirella messinensis (Claus, 1863)

Euchirella rostrata (Claus, 1866)

Euchirella truncata Esterly, 1911

Euchirella sp.

Euterpina acutifrons (Dana, 1847)

Farranula carinata Giesbrecht, 1891

Farranula rostrata (Claus, 1863)

Gaetanus kruppii Giesbrecht, 1903

Gaetanus tenuispinus (Sars G.O., 1900)

\begin{tabular}{|c|c|}
\hline \multirow{4}{*}{$\begin{array}{l}\text { CAAR } \\
\text { CABI }\end{array}$} & Oncaea mediterranea (Claus, 1863) \\
\hline & Oncaea notopus Giesbrecht, 1891 \\
\hline & Oncaea tenella Sars G.O., 1916 \\
\hline & Oncaea venusta Philippi, 1843 \\
\hline CALO & Onchocorycaeus latus (Dana, 1849) \\
\hline \multirow[t]{2}{*}{ CASI } & Onchocorycaeus ovalis (Claus, 1863) \\
\hline & Paracalanus aculeatus Giesbrecht, 1888 \\
\hline CAVA & Paracalanus denudatus Sewell, 1929 \\
\hline CEBR & Paracalanus nanus Sars G.O., 1925 \\
\hline $\mathrm{CECH}$ & Paracalanus parvus (Claus, 1863) \\
\hline \multirow[t]{2}{*}{ CEHA } & Paracalanus pygmaeus (Claus, 1863) \\
\hline & Paracartia grani Sars G.O., 1904 \\
\hline \multirow[t]{4}{*}{ CETY } & Paraeuchaeta barbata (Brady, 1883) \\
\hline & Paraeuchaeta bisinuata (Sars G.O., 1907) \\
\hline & Paraeuchaeta glacialis (Hansen, 1887) \\
\hline & Paraeuchaeta gracilis (Sars G.O., 1905) \\
\hline CLFA & Paraeuchaeta hebes (Giesbrecht, 1888) \\
\hline CLFU & Paraeuchaeta norvegica (Boeck, 1872) \\
\hline \multirow[t]{2}{*}{ CLJO } & Paraeuchaeta sarsi (Farran, 1908) \\
\hline & Paraeuchaeta tonsa (Giesbrecht, 1895) \\
\hline CLPA & Paraeuchaeta sp. \\
\hline \multirow[t]{5}{*}{ CLPE } & Paraheterorhabdus robustus (Farran, 1908) \\
\hline & Pareucalanus attenuatus (Dana, 1849) \\
\hline & Phaenna spinifera Claus, 1863 \\
\hline & Pleuromamma abdominalis (Lubbock, 1856) \\
\hline & Pleuromamma borealis Dahl F., 1893 \\
\hline \multirow{2}{*}{ COCL } & Pleuromamma gracilis Claus, 1863 \\
\hline & Pleuromamma piseki Farran, 1929 \\
\hline & Pleuromamma robusta (Dahl F., 1893) \\
\hline \multirow[t]{3}{*}{ COSP } & Pleuromamma xiphias (Giesbrecht, 1889) \\
\hline & Pontella atlantica (Milne Edwards, 1840) \\
\hline & Pontella lobiancoi (Canu, 1888) \\
\hline \multirow{3}{*}{ DIPY } & Pontella sp. \\
\hline & Pontellina plumata (Dana, 1849) \\
\hline & Pontellopsis regalis (Dana, 1849) \\
\hline \multirow[t]{2}{*}{ EUEL } & Pontellopsis villosa Brady, 1883 \\
\hline & Pseudoamallothrix ovata (Farran, 1905) \\
\hline \multirow[t]{2}{*}{ EUMA } & Pseudocalanus elongatus (Boeck, 1865) \\
\hline & Pseudocalanus minutus (Krøyer, 1845) \\
\hline EUSP & Pseudhaloptilus eurygnathus (Sars G.O. \\
\hline EUCU & 1920) \\
\hline & Rhincalanus cornutus (Dana, 1849) \\
\hline EURO & Rhincalanus nasutus Giesbrecht, 1888 \\
\hline EUTR & Sapphirina gemma Dana, 1852 \\
\hline & Sapphirina intestinata Giesbrecht, 1891 \\
\hline EUAC & Sapphirina iris Dana, 1849 \\
\hline FACA & Sapphirina lactens Giesbrecht, 1893 \\
\hline & Sapphirina nigromaculata Claus, 1863 \\
\hline & Sapphirina opalina Dana, 1849 \\
\hline GATI & Sapphirina scarlata Giesbrecht, 1891 \\
\hline
\end{tabular}

ONTE ONVE ONLA

PAAC

PADE

PANA

PAPA

PAPY

PAGR

PAGL

PAGR

PATO

PARO

PAAT

PHSP

PLAB

PLBO

PLGR

PLPI

PLRO

PLXI

PSEL

PSEU

RHCO

RHNA

SAIR

SALA

SANI

SAOP

SASC 
Goniopsyllus rostratus Brady, 1883

Haloptilus longicornis (Claus, 1863)

Haloptilus oxycephalus (Giesbrecht, 1889)

Heterocope saliens (Lilljeborg, 1863)

Heteroptilus acutilobus (Sars G.O., 1905)

Heterorhabdus abyssalis (Giesbrecht, 1889)

Heterorhabdus norvegicus (Boeck, 1872)

Heterorhabdus papilliger (Claus, 1863)

Heterorhabdus spinifrons (Claus, 1863)

Isias clavipes Boeck, 1865

Labidocera brunescens (Czerniavsky, 1868)

Labidocera pavo Giesbrecht, 1889

Labidocera wollastoni (Lubbock, 1857)

Labidocera sp.

Lubbockia squillimana Claus, 1863

Lucicutia clausi (Giesbrecht, 1889)

Lucicutia magna Wolfenden, 1903

Lucicutia flavicornis (Claus, 1863)

Lucicutia gemina Farran, 1926

Lucicutia longicornis (Giesbrecht, 1889)

Lucicutia longiserrata (Giesbrecht, 1889)

Lucicutia maxima Steuer, 1904

Lucicutia tenuicauda Sars G.O., 1907

Macrosetella gracilis (Dana, 1847)

\begin{tabular}{|c|c|c|}
\hline & Sapphirina sp. & SAAB \\
\hline & Sarsarietellus abyssalis (Sars G.O., 1905) & SCBR \\
\hline HAOX & Scaphocalanus brevicornis (Sars G.O., 1900) & SCCU \\
\hline HESA & Scaphocalanus curtus (Farran, 1926) & \\
\hline HEAC & Scaphocalanus echinatus (Farran, 1905) & SCMA \\
\hline & Scaphocalanus magnus (Scott T., 1894) & SCME \\
\hline HENO & Scaphocalanus medius (Sars G.O., 1907) & \\
\hline HEPA & Scolecithricella dentata (Giesbrecht, 1893) & \\
\hline & Scolecithricella minor (Brady, 1883) & \\
\hline ISCL & Scolecithrix bradyi Giesbrecht, 1888 & \\
\hline & Scolecithrix danae (Lubbock, 1856) & \\
\hline & Scottocalanus helenae (Lubbock, 1856) & SCPE \\
\hline & $\begin{array}{l}\text { Scottocalanus persecans (Giesbrecht 1895) } \\
\text { Subeucalanus crassus (Giesbrecht, 1888) }\end{array}$ & \\
\hline LUSQ & Subeucalanus monachus (Giesbrecht, 1888) & TELO \\
\hline & Temora longicornis Müller O.F., 1785 & TEST \\
\hline & Temora stylifera (Dana, 1849) & TRCO \\
\hline LUFL & Triconia conifera (Giesbrecht, 1891) & TRDE \\
\hline & Triconia dentipes (Giesbrecht, 1891) & TRMI \\
\hline LULO & $\begin{array}{l}\text { Triconia minuta (Giesbrecht, } 1893 \text { ["1892"]) } \\
\text { Undeuchaeta plumosa (Lubbock, 1856) }\end{array}$ & \\
\hline LUMA & Undinula vulgaris (Dana, 1849) & URFU \\
\hline LUTE & Urocorycaeus furcifer (Claus, 1863) & \\
\hline MAGR & $\begin{array}{l}\text { Valdiviella insignis Farran, } 1908 \\
\text { Xanthocalanus obtusus Farran, } 1904\end{array}$ & XАOB \\
\hline
\end{tabular}

\section{References:}

1. Alcaraz M, Calbet A, Estrada M, Marrasé C, Saiz E, Trepat I (2007) Physical control of zooplankton communities in the Catalan Sea, Prog in Oceanography, 74: 294-312.

2. Arístegui, J, Barton ED, Álvarez-Salgado X, Santo M, Figueiras F, Kifani S, Hernández- León S, Mason E, Machu E, Demarcq H (2009) Sub-regional ecosystem variability in the Canary Current upwelling. Prog. Oceanogr. 83: 33-48.

3. Auger PA, Machu E, Gorguesn T, Grima N, Waeles M (2015) Comparative study of potential transfer of natural and anthropogenic cadmium to plankton communities in the North-West African upwelling. Sci Total Envir, 505, 870-888.

4. Bainbridge V (1960) Occurrence of Calanoides carinatus (Kröyer) in the plankton of the gulf of Guinea. Nature, 188: 932-933.

5. Bainbridge V (1972) The zooplankton of the Gulf of Guinea. Bull. Mar. Ecol., 8: 61-97.

6. Barber RT, Smith RL (1981) Coastal upwelling ecosystems. In: analysis of marine ecosystems, Longhurst A (eds.), Academic Press, pp. 31-68. 
7. Barton ED (1998) Eastern boundary of the North Atlantic: northwest Africa and Iberia. In: Robinson AR, Brink KH (Eds.) The Sea. John Wiley \& Sons, pp. 633-657.

8. Belfquih M (1980) Les copépodes du plateau atlanto-marocain. Un cycle annuel dans les zones d'upwelling. PhD thesis. University of Aix-Marseille I.

9. Belmonte G, Potenza D (2001) Biogeography of the family Acartiidae (Calanoida) in the Ponto-Mediterranean Province. Hydrobiologia 453/454, 171-176.

10. Belvèze H, Erzini K (1983) The influence of hydroclimatic factors on the availability of the sardine (Sardina pilchardus Walbaum) in the Moroccan Atlantic fishery. In: Proceedings of the Expert Consultation to Examine Changes in Abundance and Species Composition of Neritic Fish Resources. San Jose, Costa Rica, April 1983. Sharp GD, Csirke J (Eds). FA.O. Fish. Rep. 291(2): 285-327.

11. Benbakhta B (1994) Structure et dynamique du zooplancton de la lagune de Moulay Bousselham (Maroc). PhD Thesis. Mohammed V University, Faculte des Sciences Rabat, 125 pp.

12. Berraho A, Ettahiri O, Brochier T, Benazzouz A, Larissi J, Makaoui A, Somoue L, Salah S, Hilmi K, Orbi A (2012) Distribution des larves de sardine et d'anchois le long du filament du Cap Ghir (région nord-ouest Africaine). J. Sci. Halieut. Aquat. 6:178-193.

13. Berraho A, Somoue L, Hernández-León S, Valdés L (2015). Zooplankton in the Canary Current Large Marine Ecosystem. In: Oceanographic and biological features in the Canary Current Large Marine Ecosystem. Valdés, L. and Déniz-González, I. (eds). IOCUNESCO, Paris. IOC Technical Series, No. 115, pp. 183-195.

14. Binet D (1988) Rôle possible d'une intensification des alizés sur les changements de répartition des sardines et sardinelles le long de la côte ouest africaine. Aquat Living Resour 1: 115-132.

15. Binet D (1991) Dynamique du plancton dans les eaux côtières ouestafricaines: écosystèmes équilibrés et déséquilibrés. Pêcheries ouest africaines, Variabilité, Instabilité et Changement (Cury P. et C. Roy, ds), ORSTOM, Paris. pp. 117-136.

16. Björnberg T (1981) Copepoda. In: Boltovskoy E (ed.). Atlas del zooplancton del Atlántico Sudoccidental. Instituto Nacional de Investigación y Desarrollo Pesquero, Mar del Plata, pp. 587-679.

17. Boltovskoy D (1999) South Atlantic Zooplankton, vols. 1-2. Leiden, Backhuys, 1706 pp.

18. Boucher J (1987) Déterminisme et dynamique de la répartition spatiale des populations de copépodes des zones de résurgences 
côtières Nord-est Atlantique et du front liguro-provençal. PhD thesis. Universuty Pierre et Marie Curie, Paris VI, 174p.

19. Boucher J (1982) Peuplement de copépodes des upwellings côtiers nord-ouest africains: Composition faunistique et structure démographique. Oceanol. Acta 5: 49-62.

20. Calbet A, Garrido S, Saiz E, Alcaraz M, Duarte CM (2001) Annual zooplankton succession in coastal NW Mediterranean waters: the importance of the smaller size fractions, J. Plankton Res. 23(3): 319331.

21. Carola M (1994) Checklist of the marine planktonic copepoda of Southern Africa and their worldwide geographic distribution. S. Afr. J. Mar. ScL, 14, 225-25.

22. Carr ME (2002) Estimation of potential productivity in Eastern Boundary Currents using remote sensing. Deep-Sea Res. II 49 : 5980.

23. Chiahou B (1997) Etude bio-écologique des copépodes pélagiques de l'estuaire atlantique du Bouregreg. $\mathrm{PhD}$ thesis. Mohammed V University, Fac. Sci. Rabat, 144 pp.

24. Chiahou B Ramdani M (1996) Observations sur les variations de la densité et de la biomasse des copépodes pélagiques des côtes atlantiques marocaines (région d'El Jadida) au cours d'un cycle annuel. Mar. Life, Vol 6 (1-2) : 21-25.

25. Chiahou B Ramdani M (1997) Systématique et distribution saisonnière des copépodes pélagiques de la côte atlantique marocaine (région d'El Jadida). J. Rech. Oceano. 22(3), 75-80.

26. Chiahou B, Ramdani M, Menioui M (1998) Structure biogéographique des copépodes des côtes marocaines, Rapport Comm. Intern. Mer. Mediterranée 2 : 406-407.

27. Coen R, Mazzocchi MG (1985) Ritrovamento di Acartia margalefi Alcaraz, 1976 (Copepoda: Calanoida) nel Golfo di Napolied in laghi costieri laziali. Oebalia 11-3, N.S.: 789-791.

28. Cropper TE, Hanna E, Bigg GR (2014) Spatial and temporal seasonal trends in coastal upwelling off Northwest Africa, 1981-2012. DeepSea Res. I 86: 94-111.

29. Cushing DH (1989) A difference in structure between ecosystems in strongly stratified waters and in those that are only weakly stratified.

J. Plankton Res. 11: 1-13.

30. David V, Sautour B, Chardy P (2007) Successful colonization of the calanoid copepod Acartia tonsa in the oligomesohaline area of the Gironde estuary (SW France)-natural or anthropogenic forcing? Estuarine, Coastal and Shelf Science 71: 429-442. 
31. De Decker A (1964) Observations on the ecology and distribution of copepoda in the marine plankton of South Africa. Dept. Comm. and Ind. Div. Sea Fisher. Pretoria, J. Inv. Rep., 49: 3-47.

32. Demarcq H, Somoue L (2015) Phytoplankton and primary productivity off Northwest Africa. In: Oceanographic and biological features in the Canary Current Large Marine Ecosystem. Valdés L, Déniz-González I (eds). IOC-UNESCO, Paris. IOC Technical Series, 115: 161-174.

33. Dickson RR, Kelly PM, Colebrook JM, Wooster WS, Cushing DH (1988) North winds and production in the eastern North Atlantic. J. Plankt. Res. 10(1):151-169.

34. El Arraj L., Tazi O., Hariss I., Hilmi K., Serghini M. and Ettahiri O. (2015) Copepods distribution patterns in an upwelling system off Northwest Africa (Southern Moroccan Atlantic coast). Int. J. of Adv. Res. V3, 6, 1136-1148.

35. Hagen E, Zülicke C, Feistel R (1996) Near-surface structures in the Cape Ghir filament off Morocco. Oceanol Acta 19, 6, 577, 598.

36. Eberwein A, Mackensen A (2006) Regional primary productivity differences off Morocco (NW-Africa) recorded by modern benthic foraminifera and their stable carbon isotopic composition. Deep Sea Res Part I. Oceanographic Research Papers 53 (8), 1379-1405.

37. Examine Changes in Abundance and Species Composition of Neritic Fish Resources. FAO Fish. Rep. 291: 731-778.

38. Flenminger A, Hulseman K (1973) Relationship of Indian Ocean Epiplanktonic Calnoides to the World Ocean. Ecological studies, Analysis and Synthesis, Heidelberg, V3.

39. Fox MH (1927) Cambridge Expedition to the Suez Canal, 1924. I: General part. Trans. zool. Soc. London 22: 1-64.

40. Furnestin ML (1957) Chaetognathes et zooplancton du secteur atlantique marocain. Rev. Trav. Inst. Pêches Marit. 21. (1 et 2).

41. Furnestin ML, Belfequih M (1976) Les copépodes du plateau continental marocain et du détroit canarien. Les espèces au cours d'un cycle annuel dans les zones d'upwelling. Cons. Int. Explor. Mer. C.M.L/9.

42. Glushko OG, Lidvanov VV (2012) Composition and Structure of the zooplankton in Coastal Waters of Mauritania in Winter. J. Sib. Fed. Univ, Biology, Vol. 2, pp. 138-150.

43. Hagen E (2001) Northwest Africain upwelling scenario. Oceanol Acta 24: 113-128.

44. Halsband-Lenk C, Hirche JH, Carlotti F (2002) Temperature effect on reproduction and development of congener copepod populations. $\mathrm{J}$ Exp Mar Biol Ecol 271:121-153. 
45. Harris RP, Wiebe PH, Lenz J, Skjodal HR, Huntley M (2000) Zooplankton methodology manual. London: Academic Press, San Diego.

46. Hernández-Guerra A, Nykjær L (1997) Sea surface temperature variability off north-west Africa: 1981-1989. int. j. remote sensing vol. 18, 12: 2539-2558.

47. Hernandez-Leon S, Gomez M, Aristegui J (2002) Mesozooplankton in the Canary Current System: The coastal-ocean transition zone. Prog. Ocean 74: 397-421.

48. Hughes P, Barton ED (1974) Physical investigations in the upwelling region of North West Africa on RRS discovery cruise 48. Tethys 6 (1-2): 43-52.

49. Larissi J, Berraho A, Makaoui A, Baibai T, Somoue L, Benazzouz A, Zizah S, Agouzouk A, Hilmi K (2013) Impact of Inter-annual Coastal Upwelling Variability (2001-2010) on the Productivity of the Moroccan Atlantic South Area $\left(21^{\circ}-26^{\circ} \mathrm{N}\right)$. Mar Biol Oceanogr. 2:1.

50. Grall JR, Laborde P, Le Corre P, Neveux J, Treguer P, Thiriot A (1974) Caractéristiques trophiques et production planctonique dans la région sud de l'Atlantique marocain. Résultats des campagnes CINECA-CHARCOT 1 et III. TETHYS 6 (1-2) pp. 11-28.

51. Jennings S, Kaiser M, Reynolds JD (2001) Marine Fisheries Ecology. Blackwell Science Ltd, Oxford. ISBN 978-0-632-05098-7.

52. Jiménez L, Lavaniegos B (2004) Changes in dominance of copepods off Baja California during the 1997-1999 El Niño and La Niña. Mar. Ecol. Prog. Ser., 277: 147-165.

53. Johnson DR, Barton ED, Hughes P, Mooers CNK (1975). Circulation in the Canary Current upwelling region off Cabo Bojador in August 1972. Deep-Sea Res 22(8): 547-558.

54. Krause M, Dippner JW, Beil J (1995) A review of hydrographic controls on the distribution of zooplankton biomass and species in the North Sea with particular reference to a survey conducted in JanuaryMarch 1987. Progr Ocean 35:81-152.

55. Lawrence D, Valiela I, Tomasky G (2004) Estuarine calanoid copepod abundance in relation to season, salinity, and land-derived nitrogen loading, Waquoit Bay, MA, Estuarine, Coastal and Shelf Science, 61: 547-557.

56. Longhurst A (1998) Ecological Geography of the Sea. Academic Press, San Diego.

57. Longhurst A, Sathyendranarth S, Platt T, Caverhill C (1995) An estimate of global primary production in the ocean from satellite radiometer data. J. of Plankton Res. Vol.17 no.6: 1245-1271. 
58. Soldevilla FL, Hernández F, Ros MM, Jiménez S, Migorance MDC, Pérez A, Consuelo de Lorenzo M (1988) Preliminary list of zooplankton of the Canary Islands. I. Cladocera, Copepoda, Euphausiacea, Chaetognatha and Salps. Bol. Mus. Mun. Funchal. 40 (196): pp 55-64.

59. Makaoui A, Orbi A, Arístegui J, Benazzouz A, Laarissi J, Agouzouk A, Hilmi K (2012) Hydrological Seasonality of cape Ghir filament in Morocco. Nat. Sci. Vol.4, No.1, 5-13.

60. Makaoui A, Orbi A, Hilmi A, Zizah S, Larissi J, Talbi T (2005) L'upwelling de la côte atlantique du Maroc entre 1994 et 1998. Compte Rendu Géoscience 337: 1518-1524 pp.7.

61. Mann KH, Lazier JRN (1991) Dynamics of marine ecosystems. Blackwell Scientific Publications, Boston, 466 pp.

62. Marcello J, Hernandez-Guerra A, Eugenio F, Fonte A (2011) Seasonal and temporal study of the northwest African upwelling system. Int. J. Remote Sens.32: 1843-1859.

63. Mauchline J (1998) The Biology of Calanoid Copepods. Academic Press, England, 710 pp.

64. Mazza J (1967) Les copepods pélagiques en Méditerranée occidentale. PhD thesis. Aix-Marseille University.

65. McGowan JA (1971) Oceanic biogeography of the Pacific. In: B.M. Funnell and W.R. Riedel (eds.). The micropaleontology of oceans. Cambridge University Press, London, pp. 3-74.

66. Meggers H, Freudenthal T, Nave S, Targarona J, Abrantes F, Helmke P (2002) Assessment of geochemical and micropaleontological sedimentary parameters as proxies of surface water properties in the Canary Islands region. Deep Sea Res. Part II: Topical Studies in Oceanography 49 (17), 3631-3654.

67. Minas HJ, Codispoti LA, Dugdale RC (1982) Nutrient and primary production in the upwelling region of Northwest Africa. Cons. Int. Explor. Mer. 180 pp.148-183.

68. Mittelstaedet E (1991) The ocean boundary along the northwest African coast: Circulation and oceanographic properties at the sea surface. Prog. Oceanog. Vol. 26, pp. 307-355.

69. Nishida S (1985) Taxonomy and distribution of the Family Oithonidae (Copepoda, Cyclopoida) in the Pacific and Indian Oceans. Bull. Ocean Res. Inst. Univ. Tokyo, 20: 1-167.

70. Nykjær L, Van Camp L (1994) Seasonal and interannual variability of coastal upwelling along northwest Africa and Portugal from 1981 to 1991. J. Gheoph. Res. 99 (C7), 14197-14207.

71. Orbi A, Hilmi K, Larissi J, Zidane H, Zizah S, El Moussaoui N, Lakhdar JI, Sarf F (1998) Hydrologie et hydrodynamique des côtes 
marocaines : milieux paraliques et zones côtières. Expo'98Lisbonne. pp : 68.

72. Ould Deddah S (1995) Modelling a multispecies schooling fishery in an upwelling environment, Mauritania, West Africa. PhD thesis. Louisiana State University, Baton Rouge, U.S.A. 78 pp.

73. Owre HB, Foyo M (1967) Copepods of the Florida current. Manuals for the identification of the fauna of the tropical Western Atlantic. Fauna caribaea: Crustacea, Part I: copepoda. Institute of Marine Science, Miami. 137 p.

74. Parrish RH, Bakun A, Husby DM, Nelson CS (1983) Comparative climatology of selected environmental processes in relation to eastern boundary current pelagic fish reproduction. In: Sharp GD, Csirke J (eds). Proceedings of the Expert Consultation to Examine Changes in Abundance and Species Composition of Neritic Fish Resources. FAO Fish. Rep., 291: 731-778.

75. Pauly D, Christensen V (1995) Primary production required to sustain global fisheries. Nature 374: 255-25.

76. Pelegrí JL, Arístegui J, Cana L, González-Dávila M, HernándezGuerra A, Hernandez-León S, Marrero-Díaz A, Montero MF, Sangrá P, Santana-Casiano M. (2005) Coupling between the open ocean and the coastal upwelling region off northwest Africa: water recirculation and offshore pumping of organic matter. J. Mar. Syst. 54 : 3-37.

77. Razouls C (1995) Diversité et répartition géographique chez les copépodes pélagiques. 1. Calanoida Annales de l'Institut Oceanographique, Paris, 71: 81-401.

78. Razouls C (1996) Diversité et répartition géographique chez les copépodes pélagiques. 2. Platycopioida, Misophrioida, Mormonilloida, Cyclopoida, Poecilostomatoida, Siphonostomatoida, Harpacticoida, Monstrilloida. Annales de 1'Institute Océanographique 71:1-149.

79. Richter D, Vink A, Zonneveld KAF, Kuhlmann H, Willems H (2007) Calcareous dinoflagellate cyst distributions in surface sediments from upwelling areas off NW Africa, and their relationships with environmental parameters of the upper water column. Mar. Micropal. 63: 201-228.

80. Rodriguez JM, Hernandez-Leon S, Barton ED (1999) Mesoscale distribution of fish larvae in relation to an upwelling filament off Northwest Africa. Deep-Sea Res. I 46: 1969-1984.

81. Salah S (2013) Structure spatio-temporelle du zooplancton dans les deux filaments d'upwelling Cap Ghir et Cap Juby. Effet de la dynamique des filaments sur la dispersion des copépodes. $\mathrm{PhD}$ thesis. Hassan II University, Fac. Sci. Ain Chock, Casablanca, 227 pp. 
82. Salah S, Ettahiri O, Berraho A, Benazzouz A, Elkalay K, Errhif A (2012) Distribution des copépodes en relation avec la dynamique du filament de Cap Ghir, (Côte atlantique du Maroc). Comptes Rendus Biologies. 335 : 155-167.

83. Salah S, Ettahiri O, Berraho A, Ramdani M, Charib S, Errhif A (2013) Diversité et structure de la communauté des copépodes dans le filament d'upwelling de cap Juby (Côte atlantique marocaine). Bull. de l'Inst. Sci. Rabat, Section Sci. de la Vie, $N^{\circ} 35$.

84. Seguin G (1966) Contribution à l'étude de la biologie du plancton de surface de la baie de Dakar (Sénégal). Étude qualitative, quantitative et observations écologiques au cours d'un cycle annuel. Bull. Inst. fr. Afr. noire, sér. A, 28 (1): 1-90.

85. Smith SL (2000) Understanding the Arabian Sea: Reflections on the 1994-1996 Arabian Sea Expedition. Deep-Sea Res. II, 48, 13851402.

86. Somoue L (2004) Structure des communautés planctoniques de l'écosystème pélagique de l'Atlantique sud marocain entre cap Boujdor et cap Blanc $\left(26^{\circ} 30^{\prime}-21^{\circ} \mathrm{N}\right)$. PhD thesis. Hassan II University, Fac. Sci. Ain Chock, Casablanca, 211 pp.

87. Somoue L, Elkhiati N, Ramdani M, Thong LH, Ettahiri O, Berraho A, Thang DC (2005) Abundance and structure of copepod communities along the Atlantic coast of southern Morocco. Acta Adr. 46 (1) 63-76.

88. Stevens I, Johnson J (2003) A numerical modelling study of upwelling filaments off the NW African coast. Oceanol. Acta 26: 549-564.

89. Susek E (2005) Environmental factors influencing cyst formation and preservation of organic-walled dinoflagellates: an environmental and laboratory study. Ph.D thesis. University of Bremen, $113 \mathrm{pp}$.

90. Thiriot A (1978) Zooplankton communities in the West African upwelling area. In: Boje R, Tomzak M (eds) Upwelling Ecosystems, New York, pp. 32-61.

91. Troupin C (2011) Study of the Cape Ghir upwelling filament using variational data analysis and regional numerical model. Ph.D thesis. University of Liège, Faculte des Sciences, 224 pp.

92. Valdés L, Moral M (1998) Time-series analysis of copepod diversity and species richness in the southern Bay of Biscay off Santander, Spain, in relation to environmental conditions. ICES J. Mar. Sci. 55: 783-792.

93. Van Camp L, Nykjaer L, Mittelstaedt E, Schlittenhardt P (1991) Upwelling and boundary circulation off Northwest Africa as depicted 
by infrared and visible satellite observations. Progr. Oceanogr., 26 (4): 357-402.

94. Verheye HM, Hutchings L, Huggett JA, Painting SJ (1992) Mesozooplankton dynamics in the Benguela ecosystem, with emphasis on the herbivorous copepods. S Afr J Mar Sci 12:561-584.

95. Verity PG, Smetacek V (1996) Organism life cycles, predation, and the structure of marine pelagic ecosystems. Mar. Ecol. Prog. Ser. 130: 277-293.

96. Vieira L, Azeiteiro U, Ré P, Pastorinho R, Marques JC, Morgado F (2003) Zooplankton distribution in a temperate estuary (Mondego estuary southern arm: Western Portugal), Acta. Oncol. 24: 163-173.

97. Wooster WS, Bakun A, McLain DR (1976) The seasonal upwelling cycle along the eastern boundary of the north Atlantic. J. Mar. Res., 34: 131-141.

98. Word Ocean Atlas 2005 Data Set (WOA 2005), National Oceanographic Data Centre, Washington D.C. Available at: http://www.nodc.noaa.gov/OC5/WOA05/pr_woa05.html.

99. Youssara F, Gaudy R, Moukrim A, Moncef M (2004) Variation spatio-temporelles du mésozooplancton de la région d'Agadir (Maroc) entre mai 1999 et décembre 2000. Mar. Life 14 (1-2) 3-18.

100. Zaafa A, Ettahiri O, Berraho A, Elkhiati N, Somoue L, Zizah S, Ramdani M, Blaghen M, Flower R (2014) A comparative study of marine zooplankton communities in the Tangier and M'Diq (Gibraltar strait) regions. Hydroécol. Appl. Tome 18, pp. 67-80.

101. Zaafa A, Ettahiri O, Elkhiati N, Blahen M, Berraho A, Somoue L, Elghrib H (2012) Variability of spatial and temporal distribution of marine zooplankton communities in relation with environmental parameters in Tangier and M'Diq (Gibraltar strait) regions. J. Mater. Environ. Sci. 3 (2) 262-269.

102. Zaafa A, Ettahiri O, Berraho A, Somoue L, Elkhiati N, Ramdani M (2010) Study of the copepod communities of Tangier and M'Diq (Atlantic and Mediterranean Moroccan coast, Strait of Gibraltar). ICES Coop. Res. Rep. N300. 76-80.

103. Zizah S, Ettahiri O, Salah S, Yahiaoui A, Ramdani M (2012) Evolution spatio-temporelle des abondances zooplanctoniques au large de la côte atlantique marocaine entre Cap Boujdour $\left(26^{\circ} 30^{\prime} \mathrm{N}\right)$ et Cap Blanc $\left(21^{\circ} \mathrm{N}\right)$. Bull. Inst. Sci. Rabat, section Sciences de la Vie, N 43 (2), 79-94. 